\title{
Geometric nonlinear diffusion filter and its application to X-ray imaging
}

\author{
Eric Michel-González, Min Hyoung Cho and Soo Yeol Lee*
}

* Correspondence: sylee01@khu.ac. $\mathrm{kr}$

Department of Biomedical

Engineering, Kyung Hee University, 1 Seocheon, Yongin, Gyeonggi,

446-701, Korea

\begin{abstract}
Background: Denoising with edge preservation is very important in digital $x$-ray imaging since it may allow us to reduce $x$-ray dose in human subjects without noticeable degradation of the image quality. In denoising filter design for $x$-ray imaging, edge preservation as well as noise reduction is of great concern not to lose detailed spatial information for accurate diagnosis. In addition to this, fast computation is also important since digital x-ray images are mostly comprised of large sized matrices.
\end{abstract}

Methods: We have developed a new denoising filter based on the nonlinear diffusion filter model. Rather than employing four directional gradients around the pixel of interest, we use geometric parameters derived from the local pixel intensity distribution in calculating the diffusion coefficients in the horizontal and vertical directions. We have tested the filter performance, including edge preservation and noise reduction, using low dose digital radiography and micro-CT images.

Results: The proposed denoising filter shows performance similar to those of nonlinear anisotropic diffusion filters (ADFs), one Perona-Malik ADF and the other Weickert's ADF in terms of edge preservation and noise reduction. However, the computation time has been greatly reduced.

Conclusions: We expect the proposed denoising filter can be greatly used for fast noise reduction particularly in low-dose x-ray imaging.

\section{Background}

Image denoising is very important in digital $\mathrm{x}$-ray imaging since it may allow us to reduce $\mathrm{x}$-ray dose in human subjects without noticeable degradation of the image quality. Owing to the advent of 2D flat-panel $\mathrm{x}$-ray detector, digital $\mathrm{x}$-ray imaging modalities like digital radiography (DR) or 3D cone-beam CT are now widely used for clinical imaging $[1,2]$. Taking $\mathrm{x}$-ray images with low $\mathrm{x}$-ray dose is always desired due to the hazardous biological effects of $x$-rays. However, low dose $x$-ray images may suffer from high level noises stemming from randomness of $\mathrm{x}$-ray photon fluence and detector noise. There have been many developments of denoising filters that can be used to improve signal-to-noise ratio (SNR) of x-ray images without compromising the image resolution to a noticeable extent [3-5]. In denoising $x$-ray images, it is critical not to lose detailed spatial information in the original images which is crucial for accurate diagnosis. Recent developments of non-linear iterative denoising filters make it possible to remove noise from the $\mathrm{x}$-ray images while maintaining edge features, which is

(c) 2011 Michel-González et al; licensee BioMed Central Ltd. This is an Open Access article distributed under the terms of the Creative Commons Attribution License (http://creativecommons.org/licenses/by/2.0), which permits unrestricted use, distribution, and reproduction in any medium, provided the original work is properly cited. 
impossible with linear filtering techniques. In implementing non-linear iterative denoising filters in $\mathrm{x}$-ray imaging, fast computation time is one of the critical factors since $\mathrm{x}$ ray images usually have large sized image matrix. DR images have big matrix sizes as large as $3500 \times 4300$ for chest imaging and $3600 \times 4800$ for breast imaging. 3D conebeam CTs based on a flat-panel detector often make images with the matrix size as large as $1024 \times 1024 \times 512$. For $x$-ray images of this big matrix size, fast computation is essential for practical use of the iterative denoising filters.

Anisotropic diffusion filter (ADF) is one of the most widely used denoising techniques in medical imaging [6-9]. ADF is believed to have good performance in denoising while well preserving edge features, and it is also often used for edge detection [10-13]. Explicit schemes of ADF needs large amount of computation [14], mostly coming from calculation of diffusion coefficients and gradients at every pixel. To improve the performance of ADF particularly in terms of computation time, we propose an iterative denoising filter considering the local image topology in the nonlinear diffusion model. We have tested the filter performance using synthetically and experimentally obtained $\mathrm{x}$-ray images. We present the performance of the proposed filter in comparison with the Perona-Malik ADF and Weickert ADF with experimental results obtained from low dose 3D micro-CT images and 2D DR images.

\section{Methods}

\section{A. Modified nonlinear diffusion filter}

Perona and Malik proposed the following partial-differential-equation (PDE) based denoising filter on a continuous domain [10]:

$$
\begin{aligned}
& \frac{\partial I}{\partial t}=\operatorname{div}[c(|\nabla I|) \cdot \nabla I] \\
& I(t=0)=I_{o}
\end{aligned}
$$

where $c(\cdot)$ is the diffusion coefficient function based on the image gradient $\nabla I$ and $I_{o}$ is the initial image. They suggested two diffusion coefficient functions of the form:

$$
c(x)=\frac{1}{1+(x / k)^{2}}
$$

and

$$
c(x)=\exp \left[-(x / k)^{2}\right]
$$

where $k$ is the noise threshold that controls the amount of diffusion to be applied in the gradient direction. Optimal choice of $k$ is known to be very important for successful denoising with the ADF and many kinds of $k$ estimators have been proposed for practical applications $[15,16]$.

An explicit discrete form of the Perona-Malik ADF is given by [10]:

$$
I_{s}^{t}(t+\Delta t)=I_{s}^{t}+\frac{\Delta t}{\left|\bar{\eta}_{s}\right|} \sum_{p \in \bar{\eta}_{s}} c\left(\left|\nabla I_{s, p}^{t}\right|\right) \nabla I_{s, p}^{t}
$$

where $I_{s}^{t}$ is the discrete image at time $t, s$ and $p$ denotes the pixel position in a discrete $2 \mathrm{D}$ grid, $\Delta t$ is the time step size in PDE, $\bar{\eta}_{s}$ is the spatial neighborhood of pixel $s$, $\left|\bar{\eta}_{s}\right|$ is the number of pixels in the window, and $\nabla I_{s, p}^{t}=I_{p}^{t}-I_{s^{\prime}}^{t}, \forall p \in \bar{\eta}_{s .}$. By implementing 
Eq. (4), calculation of the diffusion coefficient is most computationally intensive. In most discrete implementations of ADF, the number of neighborhood pixels is often chosen to be four, that is, east, west, north, and south pixels. Therefore, we need to calculate the diffusion coefficients at least four times at every pixel.

One of the limitations of this explicit discretization scheme is the stability requirement that limits the step size $\Delta t$ to less than $1 / 2 d$, where $d$ is the number of directions along which the gradients are calculated [14]. Therefore it takes longer computation time if we choose more number of directions. Despite success of the Perona-Malik model for practical use, it is known to be an ill-posed problem [10,11,17]. To overcome this problem, incorporating regularization into the PDE has been proposed $[13,18]$. In the regularized methods, the diffusion coefficients are derived from the regularized image rather than from the noisy image to avoid excessive noise effects on the gradient calculation. Other approaches, that modify the diffusion coefficients to take account of local image statistics, have been also proposed. Black and Saphiro have proposed a robust statistical estimation approach in which they used diffusion coefficients derived from the Tukey's biweight function, and they have shown better edge preservation after denoising [16]. You et al. explored a variety of anisotropic diffusion equations and they reported some improved results [17]. In all the aforementioned methods, the image features have been simply classified as $\nabla I<k$ for noise regions and $\nabla I<k$ for edge regions and its performance is well known to be successful. On the other hand, Weickert proposed a more strict anisotropic diffusion model where a diffusion tensor is used [11]. The anisotropic model mitigates the limitation of the PeronaMalik model in which the diffusion process stops at edges leaving the noises near the edges. It is believed that the Weickert model outperforms other isotropic models but it takes more extensive computation time.

In this work, we concern about the computation time of ADF in denoising medical images. We have noticed that using only a global $k$ in defining the diffusivity function works well in most cases, but more local image information must be incorporated into the diffusivity function for faster convergence. Therefore, instead of using only a global $k$ and image gradient in the diffusivity function, we are to use the global noise behavior and the local topology of the pixel intensities in defining new diffusivity functions.

Figure 1a shows a typical profile along a row in a DR image of an adult rat taken with low x-ray exposure level $(40 \mathrm{kVp}, 67 \mu \mathrm{A})$. Figure $1 \mathrm{~b}$ is the profile at the same row of the DR image taken with higher $x$-ray exposure level $(40 \mathrm{kVp}, 500 \mu \mathrm{A})$. From visual observation of Figure 1, we categorize two conspicuous types of pixels, the noise pixel and the edge pixel. A noise pixel stands for a pixel that has much higher or lower intensity than adjacent pixels having similar intensities. The edge pixel stands for a pixel that is either on an inclining slope or on a declining slope as shown in Figure 2. From the topology of the noise and edge pixels, we define the following parameters:

$$
\begin{aligned}
& D_{x}=\left\{\begin{array}{ll}
\left|I_{E}-I_{w}\right|-\delta \text { if }\left|I_{E}-I_{w}\right|>\delta, \\
0 \text { else }
\end{array},\right. \\
& A_{x}=\left(I_{E}+I_{W}\right) / 2, \\
& I_{S, x}^{\prime}= \begin{cases}I_{S}-D_{x} / 2 & I_{S}>A_{x} \\
I_{S}+D_{x} / 2 & I_{S} \leq A_{x}\end{cases}
\end{aligned}
$$



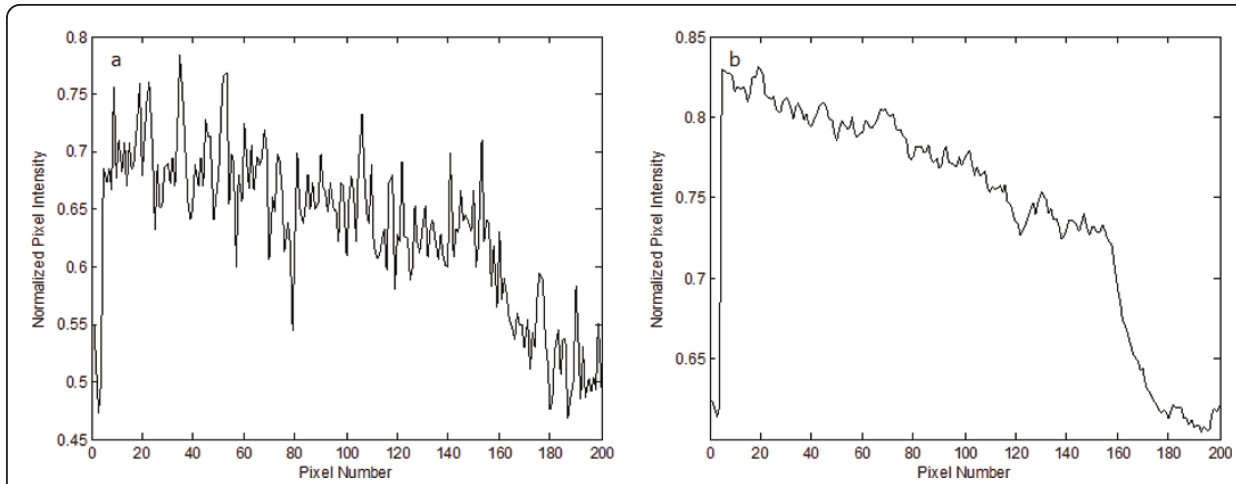

Figure 1 An $\mathbf{x}$-ray image profile. (a) A typical profile of a DR image taken with low $x$-ray exposure level. (b) The profile at the same row of the DR image taken with high $x$-ray exposure level.

$$
P_{x}=I_{S, x}^{\prime}-A_{x}
$$

where $I_{E}$ and $I_{W}$ are the image intensities at the east and west neighboring pixels, respectively, and $I_{S}$ is the image intensity of the pixel of interest. $D_{x}$ represents the $x$ directional intensity difference in a $3 \times 3$ window. $\delta \in[0, \sigma]$, where $\sigma$ is the standard deviation of the image noise, is an auxiliary parameter to prevent small noise regions from being identified as edges by setting $D_{x}$ to 0 when $\left|I_{E}-I_{W}\right| \leq \delta$. Introducing $\delta$ to $D_{x}$ has the effect of combining both global and local information on the diffusion coefficient. For automatic computation of $\delta$, we use the median absolute deviation (MAD) as suggested in [16] for calculating $k$ in ADFs and used by [6,8]. The average of the neighboring pixels in the $x$-direction, $A_{x}$, represents a local intensity reference for a given pixel. From Figure 2, we observe that $P_{x}$ is greater than $D_{x}$ at the noise-like pixel and vice versa at the edge-like pixel. We can define parameters $D_{y}, A_{y}, I_{S, y}^{\prime}$, and $P_{y}$ in the vertical direction in a similar way by considering the north and south pixels. At noise-like pixels, $P_{x}$ will have a higher value as compared to $D_{x}$ and we are to define a diffusivity function that allows fast diffusion around the noise-like pixels. Here, we define diffusivity functions that are monotonically decreasing along the $D_{x}$ or $D_{y}$

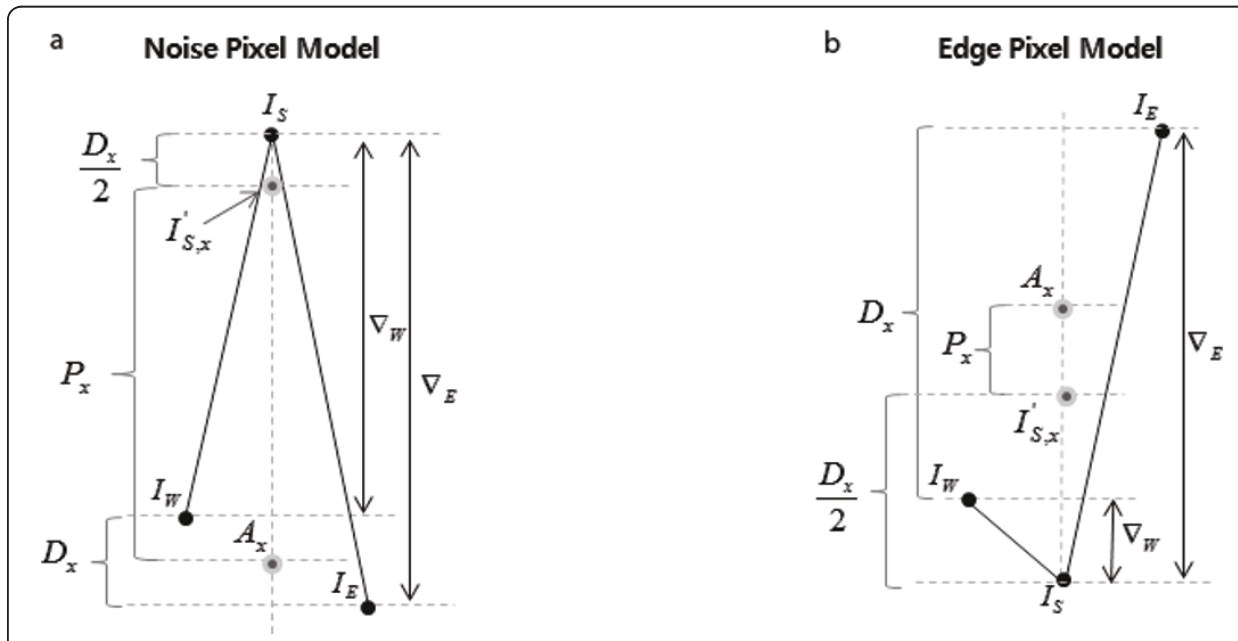

Figure 2 Pixel classifying models. (a) A typical noise pixel and (b) a typical edge pixel in the horizontal direction. $s$ is the pixel of interest and $E$ and $W$ are east-side and west-side pixels, respectively. 
direction and monotonically increasing in the $P_{x}$ or $P_{y}$ direction as below:

$$
\begin{aligned}
& c\left(D_{x}, P_{x}\right)=\frac{1}{1+\left(D_{x} / P_{x}\right)^{2}} \\
& c\left(D_{y}, P_{y}\right)=\frac{1}{1+\left(D_{y} / P_{y}\right)^{2}}
\end{aligned}
$$

At any noise pixels where $P_{x}>>D_{x} c$ becomes close to 1 allowing big diffusion. But, at the edge pixels where $P_{x}<<D_{x} c$ becomes close to 0 allowing small diffusion. As compared to the Perona-Malik diffusion coefficient which is determined by the local gradient magnitude and a global parameter $k$, the proposed diffusion coefficient is determined by the two local parameters, which results in more localized diffusion process than the original ADF. We define only one diffusion coefficient in one direction here, either horizontal or vertical, while Perona-Malik method defines two diffusion coefficients in one direction. Therefore, we can expect computation time saving by factor of two. One example of $\mathrm{c}\left(D_{x}, P_{x}\right)$ is shown in Figure 3. The discrete implementation of the proposed filter is:

$$
I_{S}^{t+\Delta t}=I_{S}^{t}+\Delta t\left[c\left(D_{x}, P_{x}\right) \cdot\left(\nabla_{E}+\nabla_{W}\right)+c\left(D_{y}, P_{y}\right) \cdot\left(\nabla_{N}+\nabla_{S}\right)\right]^{t} .
$$

where $\nabla_{p}=I_{p}-I_{S}(p=E, W, N$, and $S)$ represents the difference between the central pixel and one of the east, west, north, and south pixels respectively. Since we use the same explicit scheme used by Perona and Malik for our numerical PDE solver, the

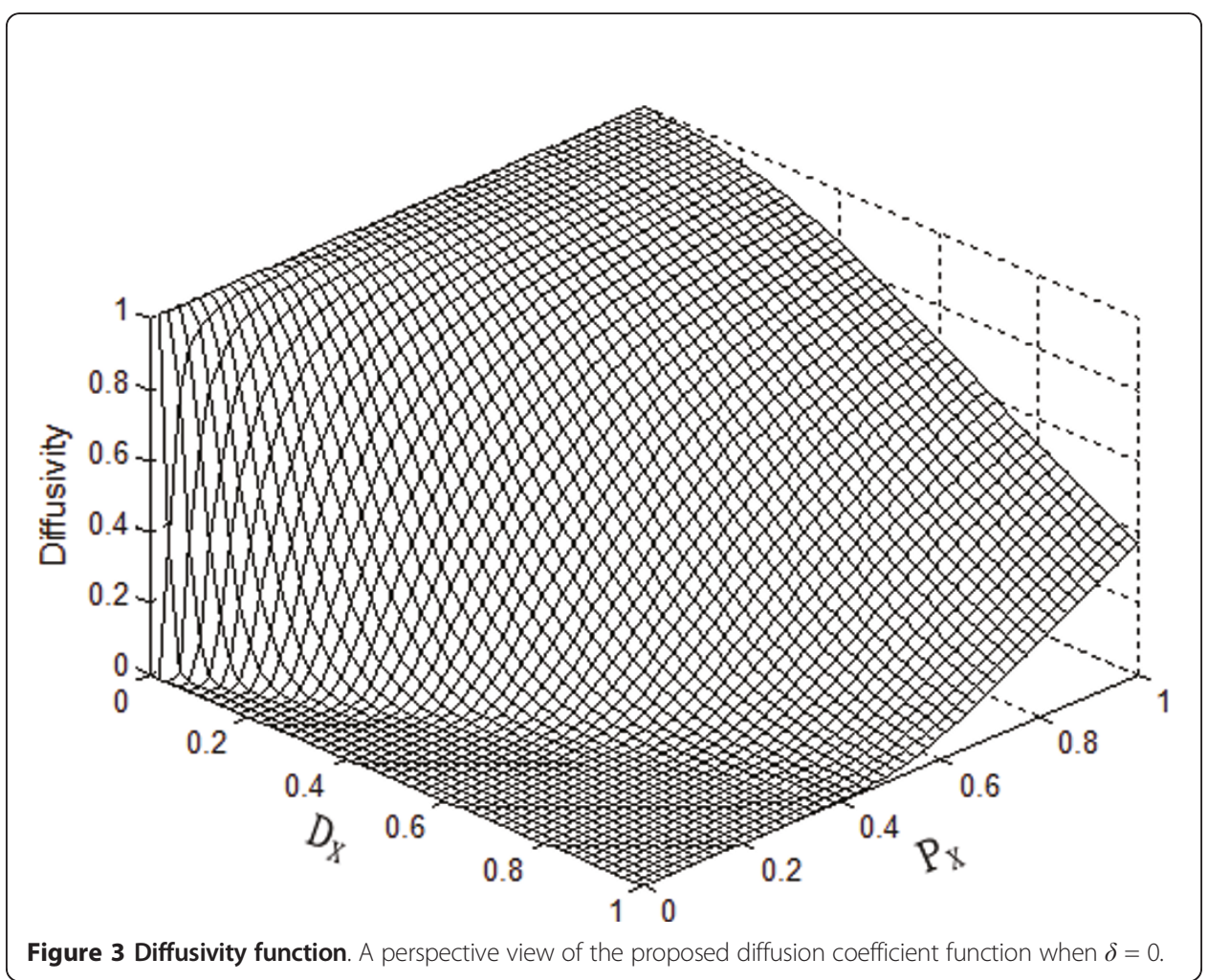


stability will depend only on the step size $\Delta t$. Previous works $[10,17]$ have pointed that the solution always converges to a steady state under a small step size (normally) $\Delta t \leq\left(\bar{\eta}_{s}\right)^{-1}$.

\section{B. Metrics for filter performance evaluation}

For quantitative evaluation of the filter performances in terms of noise reduction and edge preservation, we use the following metrics.

\section{Normalized Mean Square Error (NMSE)}

We calculate NMSE of the original and filtered images with respect to the true images when the true images are available. We assume the images taken with much higher $\mathrm{x}$ ray exposure to be the true images since they have much less noise as compared to the ones taken with low $\mathrm{x}$-ray exposure. NMSE would represent how the filtered image resembles the true image. NMSE is defined by:

$$
N M S E=\frac{\sum_{i=1}^{M} \sum_{j=1}^{N}\left(I_{f}(i, j)-I_{T}(i, j)\right)^{2}}{\sum_{i=1}^{M} \sum_{j=1}^{N}\left(I_{n}(i, j)-I_{T}(i, j)\right)^{2}} .
$$

where $I_{f}$ and $I_{n}$ are the filtered and original images, respectively, and $I_{T}$ is the true image.

\section{Noise Energy Reduction Ratio (NERR)}

Assuming the noise is white all over the spatial frequency band and the signal is mostly contained in the low spatial frequency band, we take Fourier transform of the original image and the filtered image to transform them to the spatial frequency domain. Then, we apply $2 \mathrm{D}$ radial high-pass filter with the cutoff frequency $k_{\mathrm{c}}$, that is, $H(u, v)=1$ if $\sqrt{u^{2}+v^{2}} \geq k_{c}, H(u, v)=0$ else, to the transformed images to remove the signal components residing in the low frequency band. After the high-pass filtering, $N E R R$ is then calculated by:

$$
N E R R=\frac{\sum_{i=1}^{N} \sum_{j=1}^{M}\left|D_{n}\left(u_{i}, v_{j}\right)\right|^{2}-\sum_{i=1}^{N} \sum_{j=1}^{M}\left|D_{f}\left(u_{i}, v_{j}\right)\right|^{2}}{\sum_{i=1}^{N} \sum_{j=1}^{M}\left|D_{n}\left(u_{i}, v_{j}\right)\right|^{2}}
$$

where $u$ and $v$ are the spatial frequencies in the $x$ - and $y$-directions, $D_{n}$ is the Fourier transform of the difference of the original image from the true (high $x$-ray dose) image after the high-pass filtering. $D_{f}$ is the Fourier transform of the difference of the filtered image from the true image after the high-pass filtering. The cutoff frequency $k_{\mathrm{c}}$ was chosen in each image so that most of the signal components are rejected in the calculation of NERR.

\section{Beta metric $(\beta)$}

As a measure to evaluate edge preservation and artifact formation, the beta metric $(\beta)$ is defined by [9]: 


$$
\begin{aligned}
& \beta=\frac{D\left(\Delta I_{n}-\overline{\Delta I_{n}}, \Delta I_{f}-\overline{\Delta I_{f}}\right)}{\sqrt{D\left(\Delta I_{n}-\overline{\Delta I_{n}}, \Delta I_{n}-\overline{\Delta I_{n}}\right) \cdot D\left(\Delta I_{f}-\overline{\Delta I_{f}}, \Delta I_{f}-\overline{\Delta I_{f}}\right)}}, \\
& D\left(s_{1}, s_{2}\right)=\sum_{i=1}^{M} \sum_{j=1}^{N} s_{1}(i, j) \cdot s_{2}(i, j)
\end{aligned}
$$

where $\Delta I_{n}$ and $\Delta I_{f}$ represent the edge images of the original image $I_{n}$ and the filtered image $I_{f}$, respectively. The edge images are calculated by applying the $3 \times 3$ Laplacian operator followed by thresholding to reject false edges stemming from noise. $\overline{\Delta I_{n}}$ and $\overline{\Delta I_{f}}$ are the mean intensities of $\Delta I_{n}$ and $\Delta I_{f}$, respectively. The beta metric represents the resemblance of the edge images derived from the original and filtered images. If the edge is well preserved during the denoising process, the beta metric will be close to unity.

SNR gain (g) SNR is evaluated in five different uniform-intensity ROIs with the size of $10 \times 10$. The uniform region is selected manually in each image by visual inspection. SNR in the ROI is defined by the mean pixel intensity divided by the standard deviation of the pixel intensity in the ROI. The SNR gain is defined by:

$$
g=\frac{S N R_{f}}{S N R_{n}}
$$

where $S N R_{f}$ and $S N R_{n}$ are the average SNRs in the filtered and original images, respectively.

\section{Computation time}

Computation time is evaluated on a personal computer with an Intel-Pentium ${ }^{\circledR}$ DualCore CPU E6500 @ 2.93GHz processor. The filters have been implemented using MATLAB $^{\text {TM }}$ (The Mathworks, Inc., Natic, MA.) and the computation time is evaluated excluding the data readout time from the disk to the PC memory.

\section{Results}

We first applied the proposed filter to denoising of synthetic images. As an original image, we used the Shepp-Logan head phantom image corrupted by the white
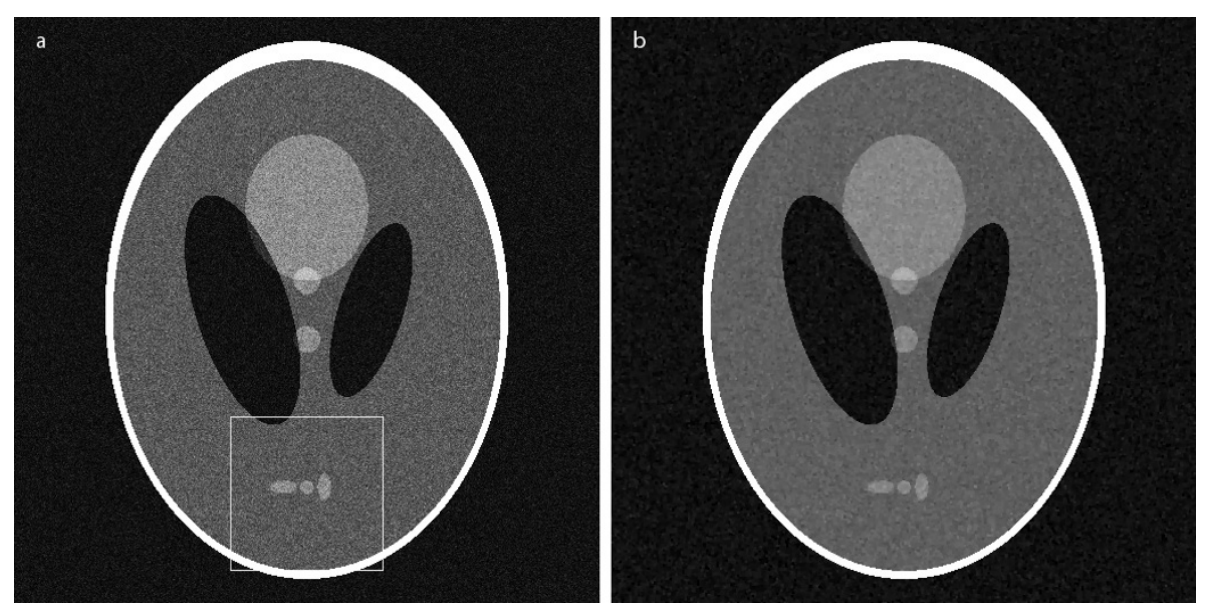

Figure 4 Filtering of a synthetic image. (a) The Shepp-Logan phantom corrupted by additive white Gaussian noise with standard deviation of 0.03 . (b) The image filtered by the proposed method. 
Gaussian noise with the standard deviation of 0.03 as shown in Figure 4a. The image filtered by the proposed method is shown in Figure 4b. The number of iterations was four and the step size in each iteration was chosen to be 0.25 according to [14]. For the sake of comparison, we also filtered the original images with two types of ADFs, one Perona-Malik ADF and the other Weickert's ADF. In both cases we used the diffusion coefficient function defined in Eq. (2) and the median regularization method suggested in [18]. Figures 5a-d show zoomed ROIs images taken from the original image, and the images filtered by the proposed method, Perona-Malik ADF and Weickert ADF, respectively. Since the Shepp-Logan phantom image has simple structures in it, it is difficult to compare the filtering effects by visual inspection. Table 1 summarizes the filter performances of the three methods. As can be seen from Table 1, the proposed method shows the noise reduction and edge preserving performances similar to Perona-Malik ADF, but the computation time of the proposed method is far less than the other two methods.

To demonstrate weak edge preservation performance, we have made a synthetic phantom shown in Figure 6a which has low contrast inclusions stained by heavy noise. We have applied the filters to remove the noise and we have shown the resulting

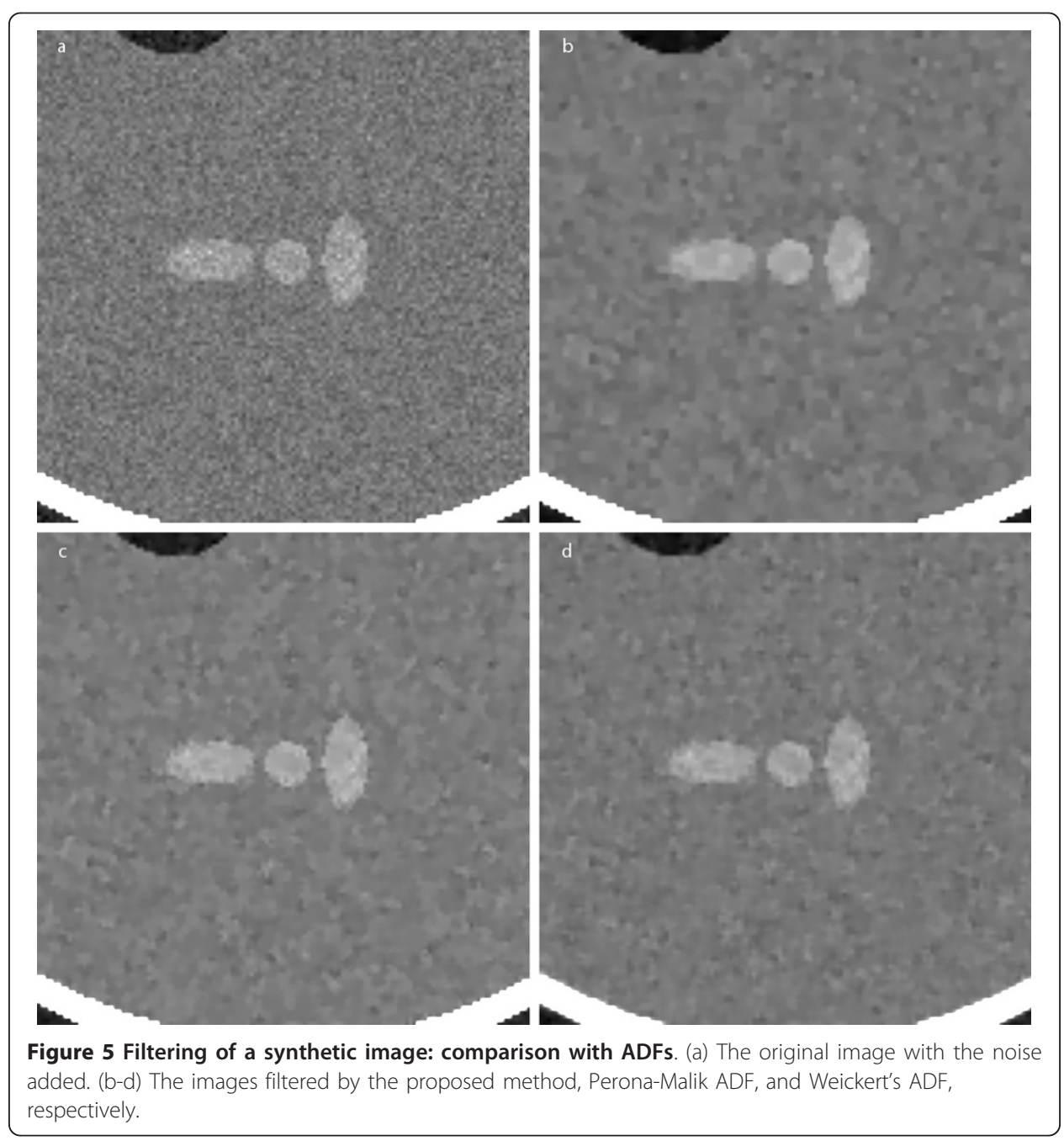


Table 1 Performance of the proposed denoising filter in comparison with other ADFs

\begin{tabular}{ccccccc}
\hline Images & Matrix size & NERR & NMSE & SNR gain & $\beta$ & Computation time (s) \\
\hline Shepp-Logan & $512 \times 512$ & 0.979 & 0.0885 & 3.40 & 0.987 & 0.428 \\
Phantom & & $(0.731)$ & $(0.364)$ & $(2.43)$ & $(0.858)$ & $(1.050)$ \\
& & {$[0.927]$} & {$[0.196]$} & {$[2.34]$} & {$[0.964]$} & {$[0.863]$} \\
\hline Mouse & \multirow{2}{*}{$240 \times 2240$} & 0.942 & 0.206 & 2.10 & 0.148 & 8.072 \\
Chest & & $(0.904)$ & $(0.393)$ & $(1.80)$ & $(0.271)$ & $(19.17)$ \\
& & {$[0.903]$} & {$[0.488]$} & {$[1.74]$} & {$[0.113]$} & {$[15.45]$} \\
\hline Micro-CT & \multirow{2}{*}{$12 \times 512$} & & & 3.46 & 0.809 & 0.484 \\
Sagittal & & & & $(2.92)$ & $(0.886)$ & $(1.26)$ \\
& & & & {$[3.26]$} & {$[0.986]$} & {$[0.781]$} \\
\hline Micro-CT & $1024 \times 1024$ & & & 2.07 & 0.894 & 1.723 \\
Coronal & & & & $(2.05)$ & $(0.914)$ & $(3.989)$ \\
& & & & {$[2.00]$} & {$[0.902]$} & {$[3.139]$} \\
\hline Micro-CT & $512 \times 512$ & & & 2.19 & 0.736 & 0.470 \\
Axial & & & & $(1.75)$ & $(0.803)$ & $(1.01)$ \\
& & & & {$[1.36]$} & {$[0.936]$} & {$[0.949]$} \\
\hline Human & \multirow{2}{*}{$3072 \times 3072$} & 0.782 & 0.376 & 1.79 & 0.163 & 12.94 \\
Chest & & $(0.778)$ & $(0.437)$ & $(1.65)$ & $(0.174)$ & $(34.69)$ \\
& & {$[0.747]$} & {$[0.470]$} & {$[1.59]$} & {$[0.166]$} & {$[26.57]$} \\
\hline
\end{tabular}

( ) represents the results obtained with Weickert ADF.

[ ] represents the results obtained with Perona-Malik ADF.

images along with the cut viewing profiles in Figure $6 \mathrm{~b}-\mathrm{d}$. The weakest contrast of the inclusions on the line is half the noise standard deviation. The proposed filter preserves the weak edges well as other ADFs do.

To test the proposed filter with real $\mathrm{x}$-ray images, we first obtained 3D micro-CT images of a sacrificed rat using a lab-built micro-CT system. During the micro-CT scan, the micro-CT acquires 2D projection images at every angle which are to be used for $3 \mathrm{D}$ image reconstruction. Therefore, any of the $2 \mathrm{D}$ projection images can be thought to be a DR image. We reconstructed 3D tomographic images using the modified Feldkamp algorithm [19]. The micro-CT system consists of a micro-focus $\mathrm{x}$-ray tube with a variable focal spot size of 5-50 $\mu \mathrm{m}$ (L8121-01, Hamamatsu, Japan), a CMOS flat-panel detector with a matrix size of $2240 \times 2240$ (C7942, Hamamatsu, Japan), a rotating subject holder for the cone-beam CT scan motion, and a data acquisition system. The pixel size of the flat-panel detector is $50 \times 50 \mu \mathrm{m}^{2}$. We performed the micro-CT scan with the tube voltage of $60 \mathrm{kVp}$ and the tube current of $340 \mu \mathrm{A}$ which gave the nominal focal spot size of $50 \mu \mathrm{m}$. The number of projection views, which determines the angular spatial resolution of the micro-CT images, was 900 . We reconstructed 3D micro-CT images with the matrix size of $512 \times 512 \times 512$ with isotropic pixel size of $170 \times 170 \times 170 \mu^{3}$ or $1024 \times 1024 \times 1024$ with isotropic pixel size of $85 \times 85 \times 85 \mu^{3}$. Since the tube current of the micro-CT is two orders of magnitude smaller than those of medical CTs, micro-CT images usually suffer from high level noise in spite of long scan time.

Figure 7a shows an example of the micro-CT images of $1024 \times 1024 \times 1024$ matrix size in coronal view and Figure 7c shows an example of the micro-CT images of $512 \times$ $512 \times 512$ matrix size in sagittal view. Since the micro-CT images are in full 3D format, we can view the images in arbitrary direction. Figures $7 \mathrm{~b}$ and $7 \mathrm{~d}$ show the images filtered by the proposed method in four iterations. For better visualization of this images, magnified version of the ROIs are shown in Figure 8 and Figure 9. Here, we have also shown the images filtered by the two ADFs in four iterations and the same 

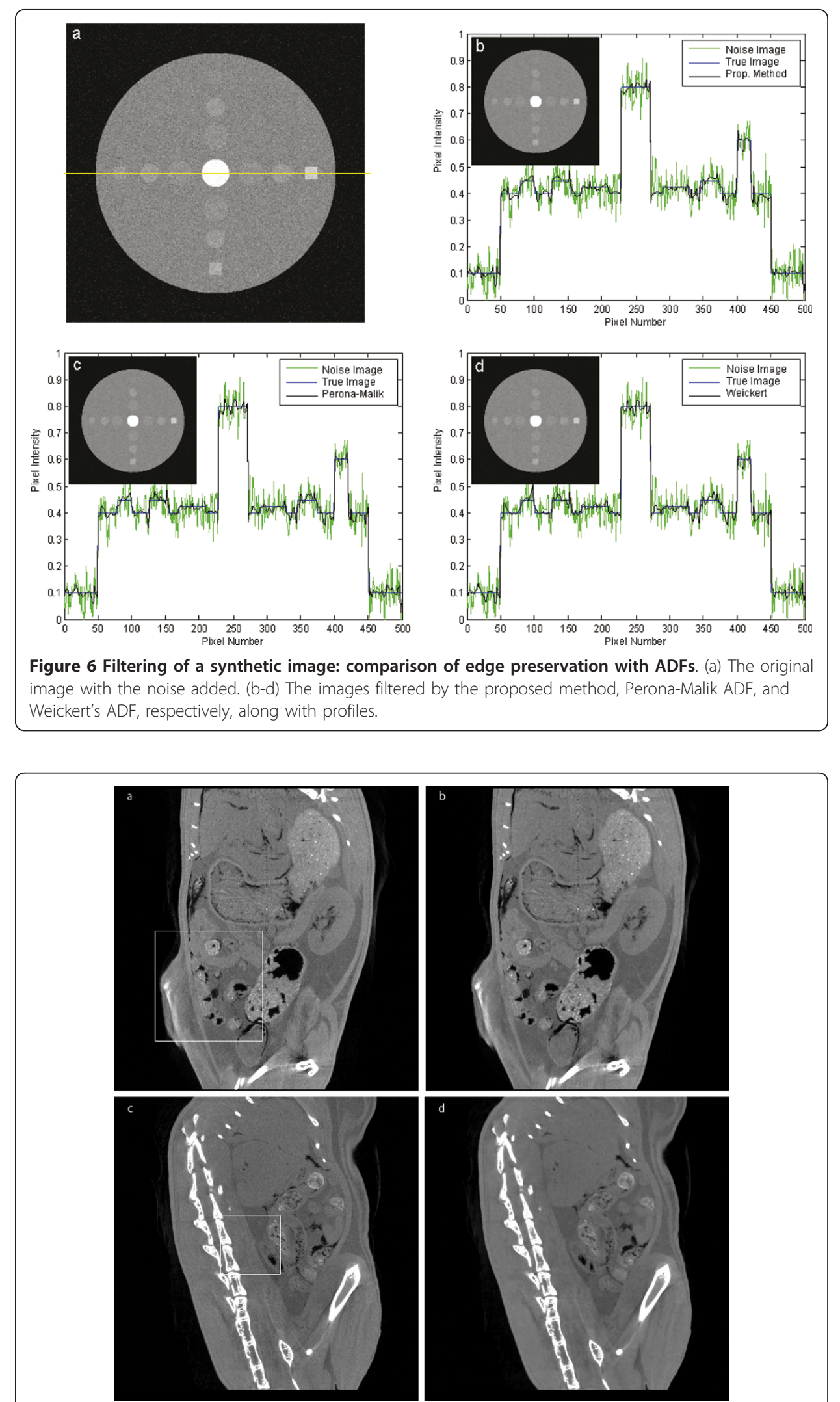

Figure 7 Filtering of micro-CT images of a rat. (a) A coronal image before filtering and (b) after filtering. (c) A sagittal image before filtering and (d) after filtering. The square boxes are the region of interest. 

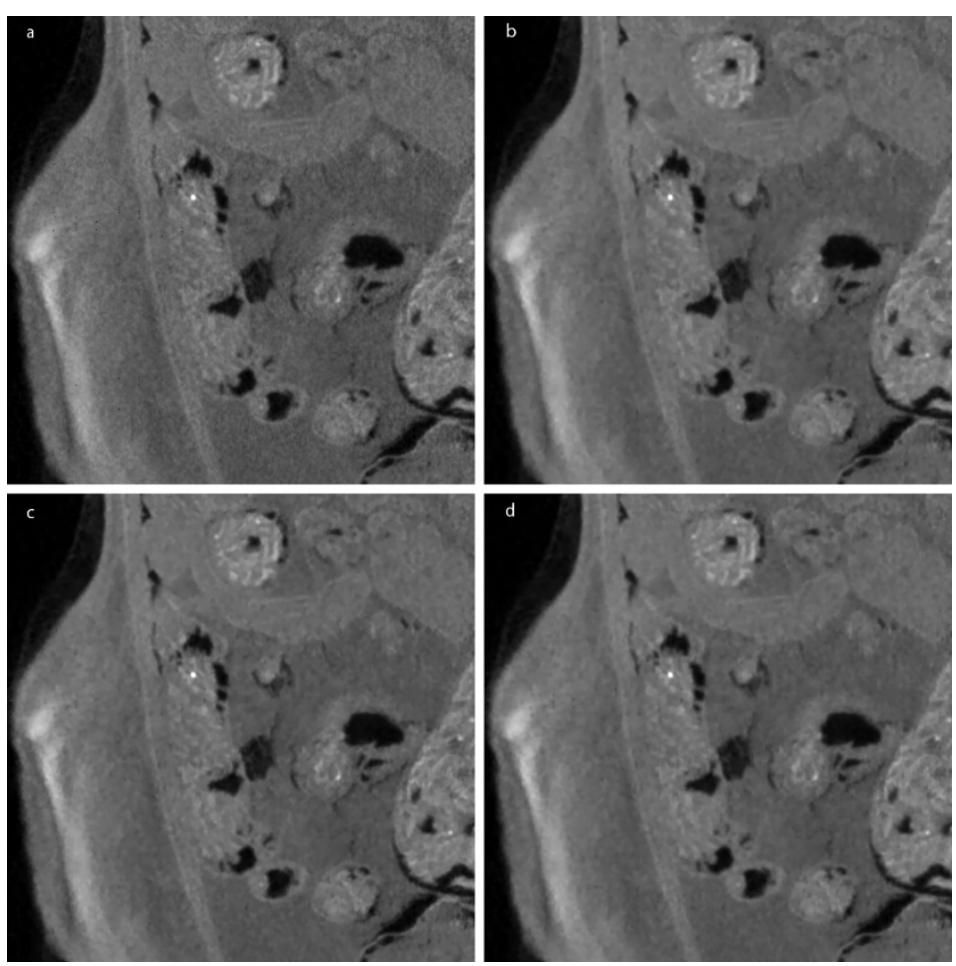

Figure 8 Filtering of micro-CT images of a rat: zoomed coronal views. (a) The original image. (b-d) The images filtered by the proposed method, Perona-Malik ADF, and Weickert's ADF, respectively.
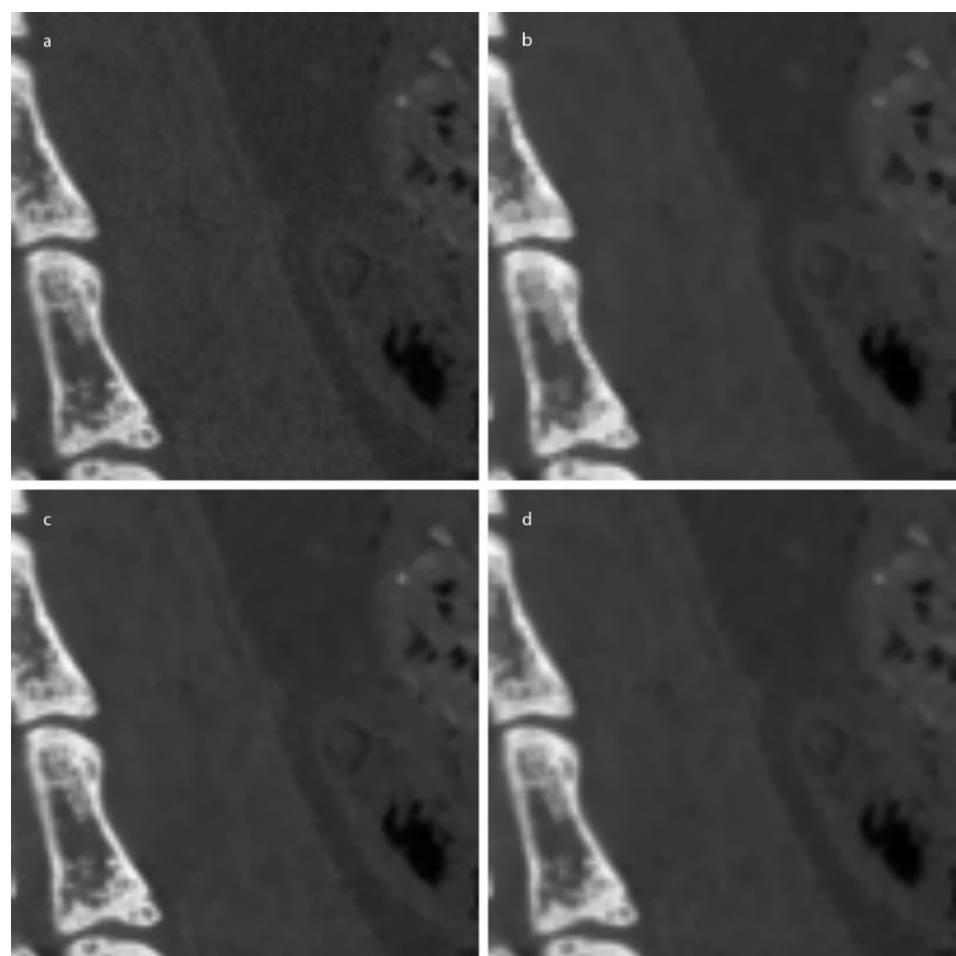

Figure 9 Filtering of micro-CT images of a rat: zoomed sagittal views. (a) The original image. (b-d) The images filtered by the proposed method, Perona-Malik ADF, and Weickert's ADF, respectively. 
time step size of 0.25 . As can be seen from Figure 8 and 9, the proposed filter well removes the noise while preserving small anatomic structures. Figure 10a shows a DR image of an adult rat taken with the tube voltage of $40 \mathrm{kVp}$ and the tube current of 67 $\mu \mathrm{A}$. The exposure time was $1 \mathrm{~s}$. The image matrix size is $2240 \times 2240$ with the pixel size of $50 \times 50 \mu \mathrm{m}^{2}$. Figure 10c shows a magnified version of the ROI indicated by the white rectangle in Figure 10a. Figures $10 \mathrm{~b}$ and $10 \mathrm{~d}$ show the images filtered by the proposed method.

Figure 11a shows the sagittal micro-CT image on which salt and pepper noises have been intentionally added. Salt and pepper noises may appear on DR images due to the defective detector elements. Figures 11b-d show the images filtered by the proposed filter and the two ADFs, respectively. Both the ADFs do not remove the salt and pepper noises completely whilst the proposed filter removes the noises very well.

Table 1 summarizes the filter performances in all the cases shown in the previous figures and also in extra cases of an axial-view micro-CT image of the rat and a DR image of a human chest. In the case of human chest imaging, we took the true image with much higher tube current of $2.2 \mathrm{~mA}$ as compared to $0.4 \mathrm{~mA}$ in normal imaging. The tube voltage was $104 \mathrm{kVp}$. As can be noticed from Table 1, the overall performance of the proposed filter is similar to those of the two ADFs in terms of noise

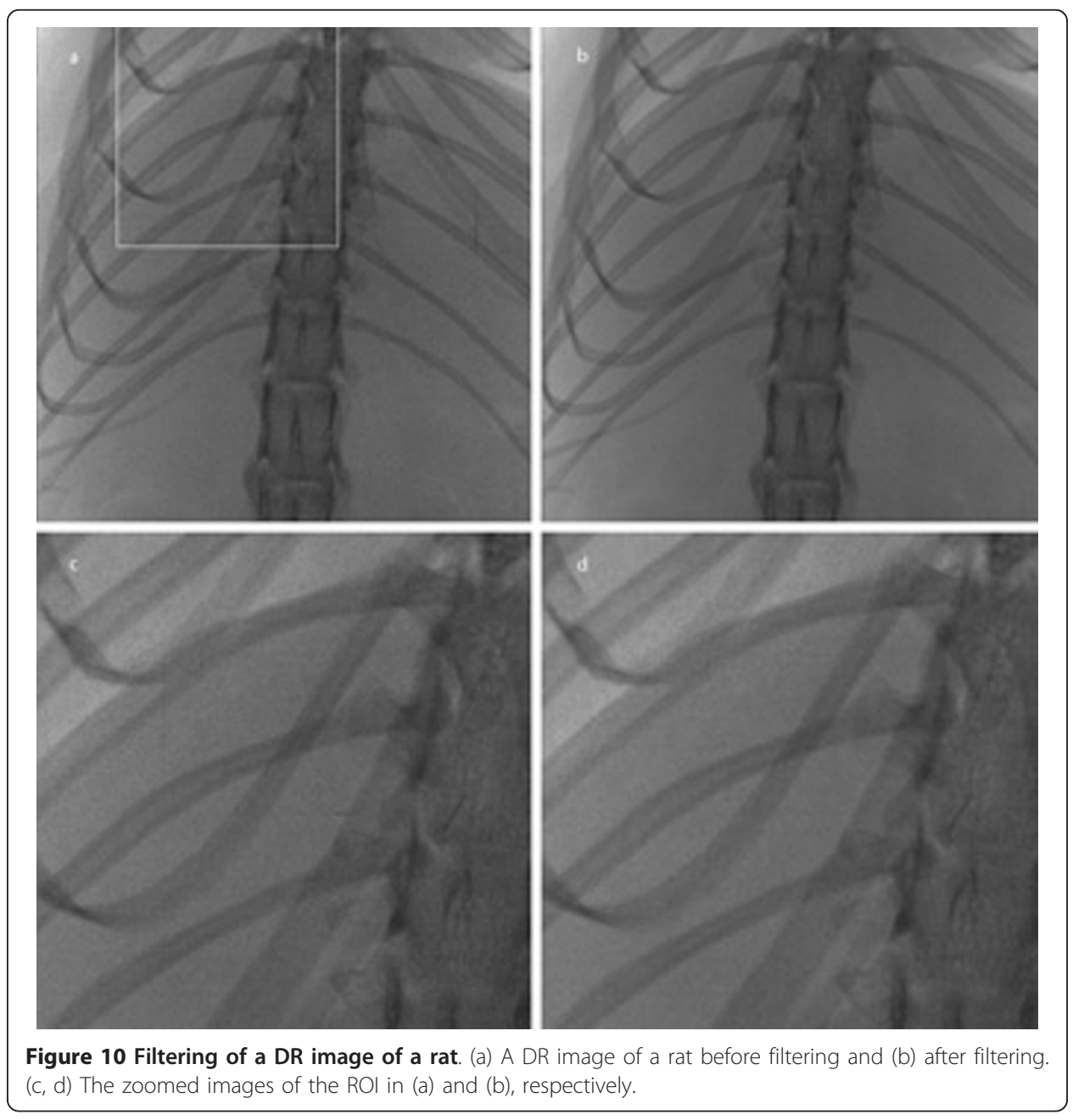




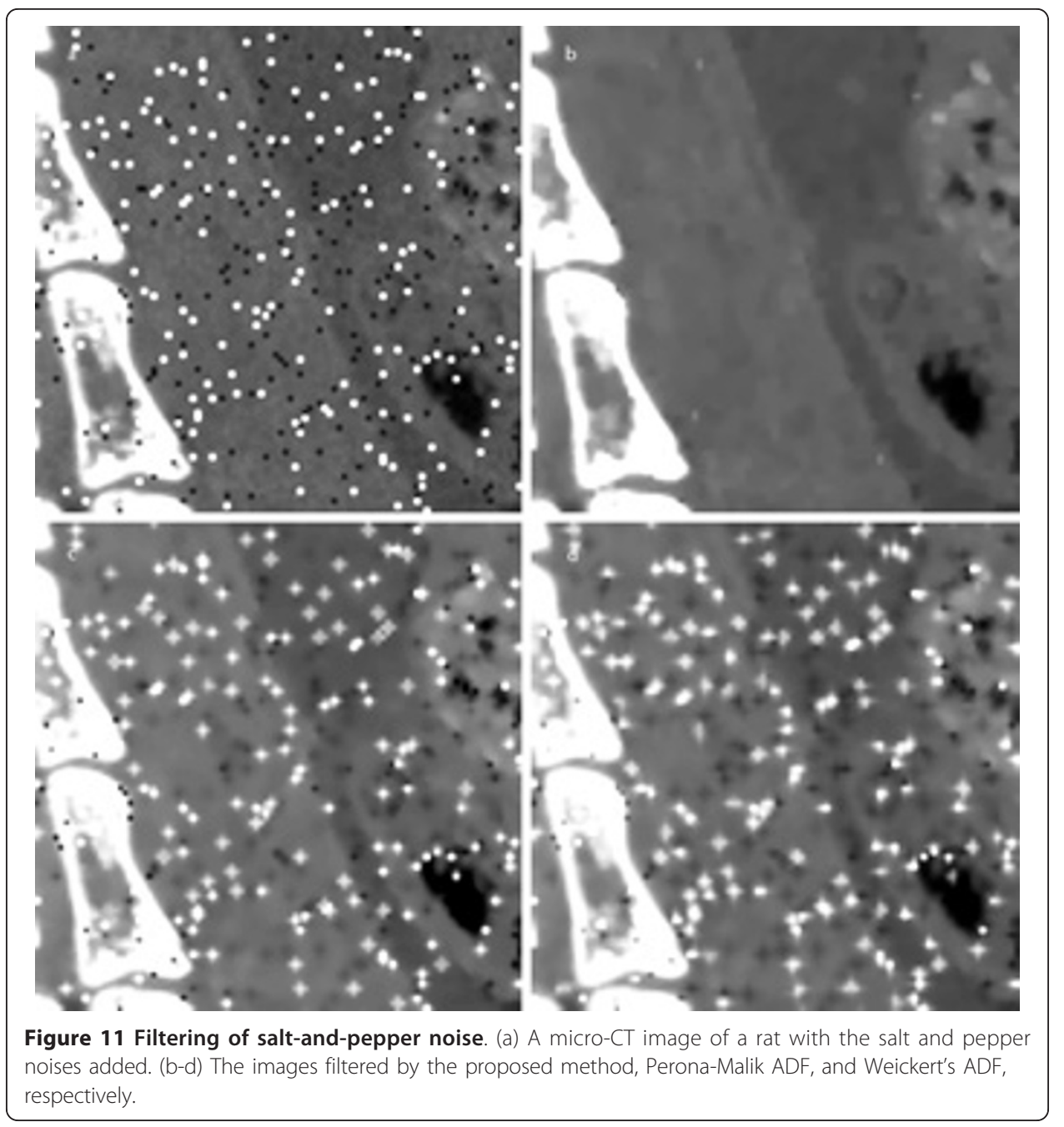

reduction and edge preservation. But, the computation time of the proposed filter is much shorter than the two ADFs.

\section{Discussion}

The proposed filter uses the same form of PDE equation as the Perona-Malik ADF but with a different way of diffusion coefficient calculation. In the original Perona-Malik ADF, four different diffusion coefficients are employed to a given pixel in four orthogonal directions to allow different amount of diffusion in each direction according to the gradient strength. Owing to the four directional diffusion processes, the PeronaMalik ADF has better edge preservation than a linear diffusion filter. Even though the proposed filter uses only two diffusion coefficients in solving the PDE, it turns out that the proposed filter has similar performance to the Perona-Malik ADF. In Perona-Malik $\mathrm{ADF}$, regularization must be incorporated into the diffusion coefficient calculation to stabilize the diffusion process avoiding the influence of high noises. Since the proposed filter is not based on the gradient-dependent diffusivity function with a global noise threshold, high impulsive noises do not affect the performance of the proposed filter as can be seen from the salt-and-pepper noise case in Figure 11. Even without any regularization in the proposed filter, we have not observed any unstable operations either 
in denoising the images we have used so far. Filtering without regularization has also allowed further reduction of computation time of the proposed filter. It is believed that the Weickert's ADF better removes the noise at the edge region, but it takes much longer computation time. Although fast algorithms to solve the Weickert's ADF have been proposed, those schemes suffer from bigger errors and artifacts [14]. There are also many other iterative non-linear denoising filters than ADFs, which are thought to outperform ADFs in many respects [20-22].

Stopping iterations at the right time during the diffusion filtering process is an important issue. Weickert introduced a stopping criterion based on the variance of the filtered image relative to the variance of the original image [23]. Later, Mrázek proposed the so-called decorrelation criterion which minimizes the covariance of the image to the noise [24]. Bazan observed that the correlation between $I(t)$ and $I(0)$ decreases gradually from 1 to 0 as $t \rightarrow \propto$, and suggested that the iteration be stopped when the second derivative of correlation between $I(t)$ and $I(0)$ reaches a maximum that is very close to the correlation between the true and the filtered image [25]. We have found that Bazan's criterion agrees well with the behavior of the proposed filter. Figure 12 shows the NMSEs of the three filters calculated at every iteration step during the filtering of the rat image shown in Figure 10. It demonstrates that as the filtering process goes by, the proposed filter also converges to a steady state as the other methods. Even though the steady state error of the proposed filter is greater than the other methods, the proposed filter converges faster in the early stage of iterations. Based on the Bazan's criterion, the optimal number of iterations was four for this case which is smaller than those of the other filters. For other cases, the optimal number of

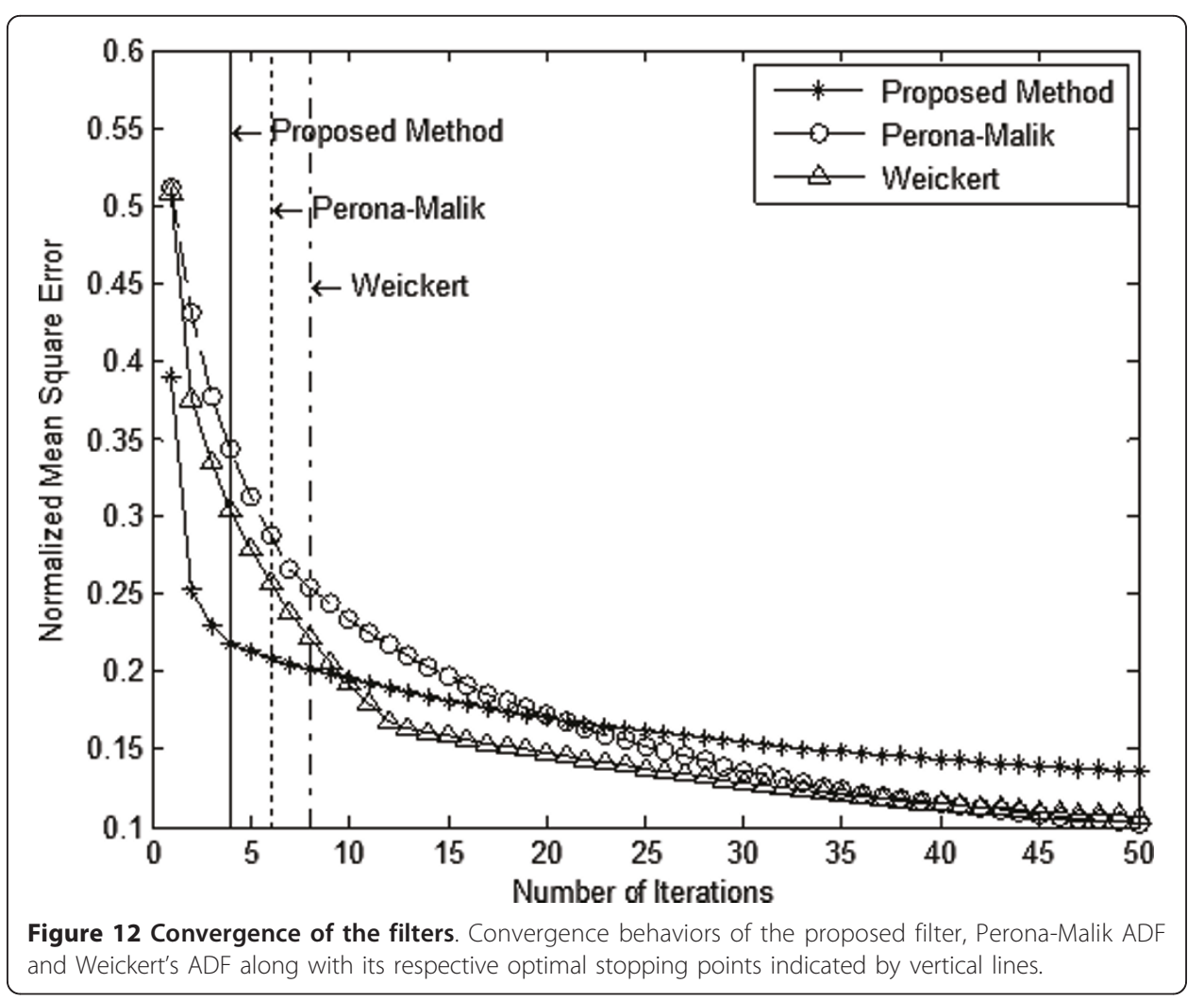


iterations for the proposed filter ranges from 4 to 6 which always are smaller than those of the other filters. Until the iteration reaches the optimal point, the NMSE of the proposed filter is smaller than those of the other filters. We think faster convergence of the proposed filter is due to the use of local pixel topology in defining the diffusion coefficient, which makes the filter more robust to high intensity noises.

\section{Conclusions}

We have experimentally confirmed that the proposed filter and the two ADFs have similar filter performance in terms of noise reduction and edge preservation. However, the proposed filter takes much shorter computation time than the two ADFs do. We expect the proposed filter can be greatly used to mitigate the noise effects in low-dose $x$-ray imaging.

Acknowledgements

This work was supported by the National Research Foundation (NRF) grant funded by the Korean government (MEST) (No: 2009-0078310)

\section{Authors' contributions}

EM has devised the new filter design structure and implemented the proposed filter as well as the conventional ADFs using MATLAB ${ }^{\mathrm{TM}}$. He has also evaluated the filter performances using the synthetic and experimentally images. MH has guided EM in how to evaluate the filter performances. SY has obtained micro-CT images and DR images and he has also drafted and finalized the manuscript. All authors have read and approved the final manuscript.

\section{Competing interests}

The authors declare that they have no competing interests.

Received: 15 February 2011 Accepted: 5 June 2011 Published: 5 June 2011

\section{References}

1. Ning R, Chen B, Yu R, Conover D, Tang X, Ning Y: Flat panel detector-based cone-beam volume CT angiography imaging: system evaluation. IEEE Trans Med Imag 2000, , 19: 949-963.

2. Lee SC, Kim HK, Chun IK, Cho MH, Lee SY, Cho MH: A flat-panel detector based micro-CT system: performance evaluation for small-animal imaging. Phys Med Biol 2003, , 48: 4173-4185.

3. Ferrari RJ, Winsor R: Digital radiographic image denoising via wavelet-based hidden Markov model estimation. Journal of Digital Imaging 2005, 18:154-167.

4. Zhong J, Ning R, Conover D: Image denoising based on multiscale singularity detection for cone beam CT breast imaging. IEEE Trans Medical Imaging 2004, 23:696-703.

5. Hensel M, Pralow T, Grigat RR: Real-time denoising of medical x-ray image sequence: three entirely different approaches. LNCS 2006, 4142:479-490.

6. Yongjian Y, Acton ST: Speckle reducing anisotropic diffusion. IEEE Trans Image Process 2002, 11:1260-1270.

7. Zhang F, Yoo YM, Koh LM, Kim Y: Nonlinear diffusion in Laplacian pyramid domain for ultrasonic speckle reduction. IEEE Trans Medical Imaging 2007, 26:200-211.

8. Mittal D, Kumar V, Saxena SC, Khandelwal N, Kalra N: Enhancement of the ultrasound images by modified anisotropic diffusion method. Med Biol Eng Comput 2010, 48:1281-1291.

9. Gerig G, Kubler O, Kikinis R, Jolesz FA: Nonlinear anisotropic filtering of MRI data. IEEE Trans Medical Imaging 1992, 11:221-232.

10. Perona P, Malik J: Scale-space and edge detection using anisotropic diffusion. IEEE Trans Pattern Anal Machine Intell 1990, 12:629-639

11. Weickert J: Anisotropic Diffusion in Image Processing. In European Consortium for Mathematics in Industry Edited by: Stuttgart, BG Teubner 1998, 14-26.

12. Weickert J: A review of nonlinear diffusion filtering. In Lecture Notes in Computer Science, Scale-Space Theory in Computer Vision Edited by: ter Haar Romeny BM, Florack L, Koenderink J, Viergever M Berlin, Springer-Verlag 1997 1252:3-28

13. Catté F, Lions PL, Morel JM, Coll T: Image selective smoothing and edge detection by nonlinear diffusion. SIAM Journal on Numerical Analysis 1992, 29:182-193.

14. Weickert J, Romeny BM, Viergever MA: Efficient and reliable schemes for nonlinear diffusion filtering. IEEE Transactions on Image Processing 1998, 7:398-410.

15. Canny J: A computational approach to edge detection. IEEE Trans Pattern Anal Machine Intell 1986, 8:679-698.

16. Black MJ, Sapiro G, Marimont D, Heeger D: Robust anisotropic diffusion. IEEE Trans Image Process 1998, 7:421-432.

17. You Y, Xu W, Tannenbaum A, Kaveh M: Behavioral analysis of anisotropic diffusion approach to edge detection. IEEE Trans Image Process 1996, 5:1539-1552.

18. Demirkaya O: Smoothing impulsive noise using nonlinear diffusion filtering. CVAMIA-MMBIA, LNCS 2004, 3117:111-122.

19. Feldkamp LA, Davis LC, Kress JW: Practical cone-beam algorithm. J Opt Soc Am 1984, 1:612-619. 
20. Rudin LI, Osher S, Fatemi E: Nonlinear total variation based noise removal algorithms. Physica D 1992, 60:259-268.

21. Chambolle A, Lions PL: Image recovery via total variation minimization and related problems. Numerische Mathematik 1997, 76:167-188.

22. Sattar F, Floreby L, Salomonsson G, Lovstrom B: Image enhancement based on a nonlinear multiscale method. IEEE Trans Image Process 1997, 6:888-895

23. Weickert J: Coherence-enhancing diffusion of colour images. Image and Vision Computing 1999, , 17: 201-212.

24. Mrázek P, Navara M: Selection of optimal stopping time for nonlinear diffusion filtering. International Journal of Computer Vision 2003, 52:189-203.

25. Bazan C, Blomgren P: Image smoothing and edge detection by nonlinear diffusion and bilateral filter.[http://www. csrc.sdsu.edu/csrc/acsess/reports/ACSESS200806.pdf].

doi:10.1186/1475-925X-10-47

Cite this article as: Michel-González et al: Geometric nonlinear diffusion filter and its application to X-ray imaging. BioMedical Engineering OnLine 2011 10:47.

Submit your next manuscript to BioMed Central and take full advantage of:

- Convenient online submission

- Thorough peer review

- No space constraints or color figure charges

- Immediate publication on acceptance

- Inclusion in PubMed, CAS, Scopus and Google Scholar

- Research which is freely available for redistribution 